\title{
Prediction of traffic flow based on the EMD and wavelet neural network Teng Feng ${ }^{1, a}$,Xiaohong Wang ${ }^{1, b}$, Yunlai $\mathrm{He}^{1, \mathrm{c}}$ \\ ${ }^{1}$ Electronic and Control Engineering College, Chang'an University, Xi'an 710064, China \\ auserfengt@163.com, bwxh429497419@163.com, cheyunlai1991@163.com
}

Keywords: traffic flow; prediction; EMD; wavelet neural network

\begin{abstract}
The operation of the urban traffic exist a high degree of complexity and randomness. The key of Intelligent Transportation System is the real-time and accurate traffic flow prediction. Taking effective measures in a timely manner would prevent the occurrence of traffic accidents since traffic congestion brings much traffic inconvenience to people. Real-time traffic flow prediction plays a significant role in easing traffic congestion and guiding convenient travelling. Therefore, considering the characteristics of traffic flow, this paper presents a neural network, wavelet analysis method, and the EMD and wavelet neural network method respectively. Three methods are utilized in simulating the same set of traffic data and then the most effective way of solving traffic congestion is obtained by taking contrast analysis of simulation result.
\end{abstract}

\section{Introduction}

With the rapid development of social economy, and on the basis of existing infrastructure, the increasing of all kinds of vehicle ownership has made serious traffic jams, which lead to accidents frequently. Therefore, the way of systemly considering vehicles and roads is the key of the problem. The prediction of the corresponding road is acquired by analyzing and processing the data through related algorithm after investigating the situation of highway traffic especially investigating peak of road traffic flow, grasping specific characteristics of traffic flow, traffic flow distribution, traffic composition and vehicle running speed effectively. Consequently, drivers would obtain the optimal route for travelling.

A wide range of traffic flow forecasting model can be divided into model based on statistical theory, model based on the theory of nonlinear prediction, model based on the theory of the neural network, model based on the theory of dynamic allocation and model based on microscopic traffic simulation and so on. The neural network [1] is the most widely used one among the mentioned models. Though the neuronal structure and internal transfer function of traditional neural network is simple and it has good ability in dealing with information, however, the entire network information storage capacity is limited, which leads to its optimal speed slow, low real-time performance and generalization ability.

In practice work of prediction, different forecast methods can be used to establish kinds of prediction model. Different prediction methods comes from various angles analyze useful information from different aspects. It is difficult to obtain the real model to reflect the actual process because of [2] the uncertain and changing of the prediction environment. Meanwhile due to the interaction of different influence factors in the environment of prediction, the actual process will be changed in unexpected way and thus the single forecasting model for some factors will face the risk of error hypothetical.

\section{Methods and Principles}

Wavelet Neural Network. Wavelet neural network based on wavelet transform and artificial neural network has been widely used in [3] signal processing, data compression, fault diagnosis and etc. The wavelet neural network has the optimal ability of function approximation. The common inherent defects of artificial neural network model could be overcame effectively due to the difference its algorithm in comparison with ordinary model of neural network. Simultaneously, better prediction could be acquired according to the established prediction model. Wavelet neural 
network structure is showed in Figure 1. $x_{1}, x_{2}, \ldots, x_{m}$ is wavelet neural network input parameters, $y_{1}, y_{2}, \ldots y_{m}$ is prediction of wavelet neural network output.

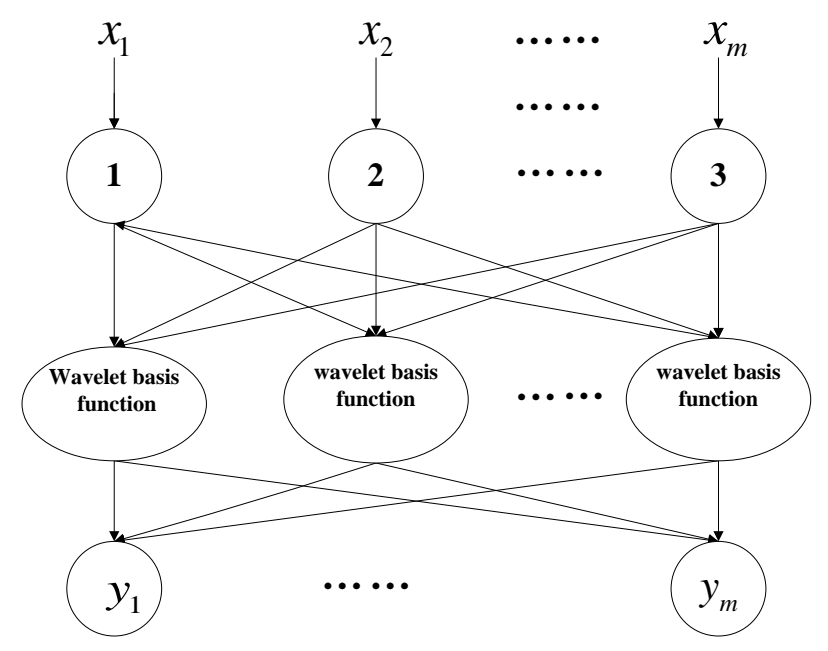

Fig. 1 Wavelet neural network structure

Wavelet neural network based on the theory of wavelet analysis is a new branch in the study of neural network in recent years. Wavelet neural network which is highly nonlinear and good local characteristics makes up the deficiency of the traditional BP network in convergence and timeliness. The wavelet neural network is utilized in traffic flow prediction due to its great performance in nonlinear approximation and nonlinear parameter estimation. This paper applies the wavelet neural network in real-time forecasting of urban traffic flow for the sake of improve the efficiency of the prediction model.

Empirical Mode Decomposition. Under interference of themselves' and external factors, shortterm traffic flow is a kind of typical non stationary signal that has complicated data elements. Its different [4] signal components have various characteristics which make different influence in the trends of traffic flow. Deterministic signal determines the overall trend of the change of traffic flow while uncertain disturbance signals make the actual traffic flow changing around the trend line. Empirical Mode Decomposition (EMD) is a kind of new method for processing non-linear and nonstationary signal. Unstable signal is decomposed into several Intrinsic Mode components step by step according to the fluctuation scales and trend. Each component with different bandwidths contains the signal from high frequency to low and the EMD method is adaptive decomposition according to the inherent characteristics of the signal. Therefore, all components can reflect the essence and potential regulation of the traffic flow. The theory related to EMD is used to decompose the actual traffic flow signal in the experiment. After removing the jamming signal, the wavelet neural network prediction models are established by considering each useful signal component and then all predicted values are added to the final result.

The specific algorithm is as follow:

(1) use EMD decompose the traffic flow data;

(2) analyse the intrinsic mode components and remove the random disturbance components in traffic flow;

(3) uniformize processing useful component;

(4) establish the wavelet neural network prediction models using useful signal components;

(5) normalize the forecast results;

(6) add all predicted data to the final traffic flow prediction result. 


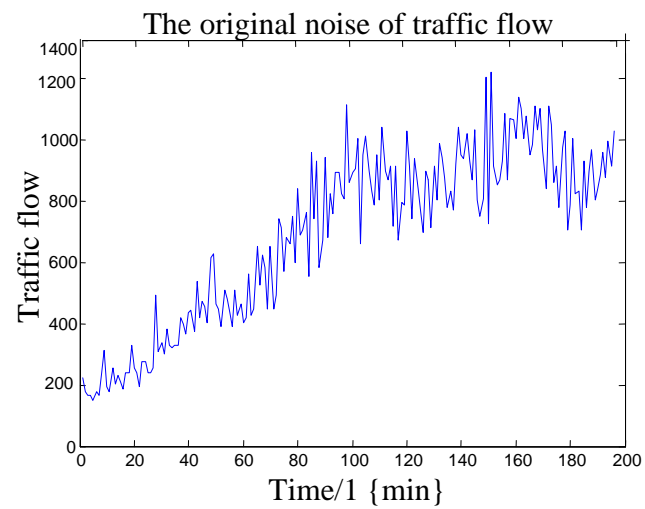

Fig. 2 The noisy traffic flow collected

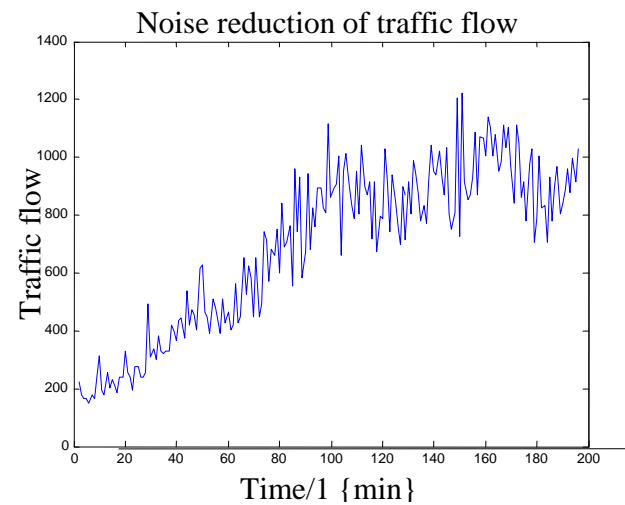

Fig. 3 Traffic flow of noise reduction

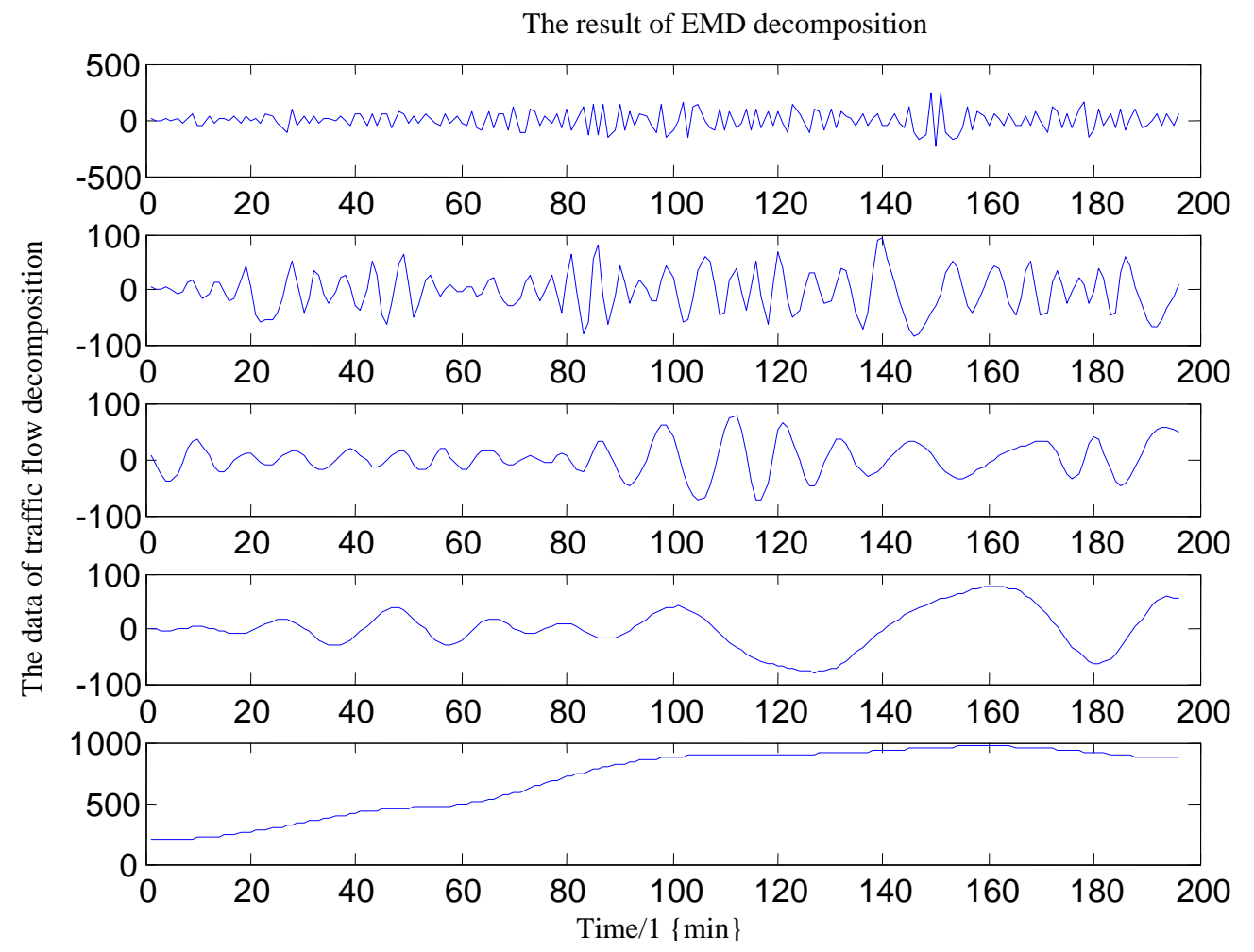

Fig. 4 The result of traffic flow of EMD decomposition

According to the proposed prediction algorithm EMD method is employed to decompose the collection of traffic flow data. Figure 2 illustrates the noise of traffic flow in the first day while figure 4 showed the decomposition result. It is obvious that the non-stationary of each part of traffic flow is reduced and the first two modal components are mainly random disturbance. Thus $[5,6]$ it has been removed directly during the forecast so that the reduction of noise of traffic flow data is achieved. The result of noise reduction is shown in figure 3 . The network consists of the input, hidden and output layers which have 4, 6 and 1 neurons respectively. Input neurons evince the first four forecast time node of traffic flow while the output neurons indicate the prediction of the traffic flow. Finally the predictive results of the modal of the effective signal are accumulated and the eventual prediction is obtained.

\section{The instance simulation and analysis}

Firstly, the traffic data of four days is collected and the traffic flow is recorded every 15 minutes within this period. Then the wavelet neural network which would be used for testing the traffic flow in the fourth day is trained by the data in first three days. Finally the tested traffic flow is compared with the actual data. 
The method mentioned above is utilized to predict the traffic flow and the predicted results is analyzed with the evaluation index. The traffic flow data of prediction are the same.

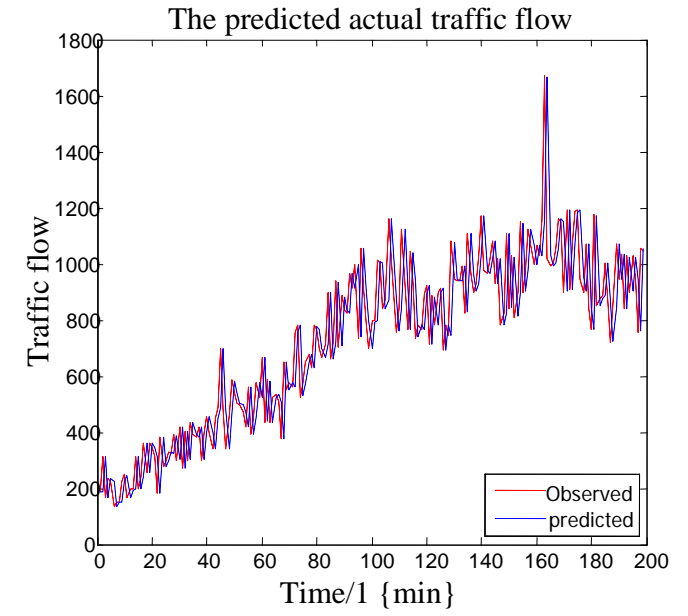

Fig. 5 Single-index moving predicted results

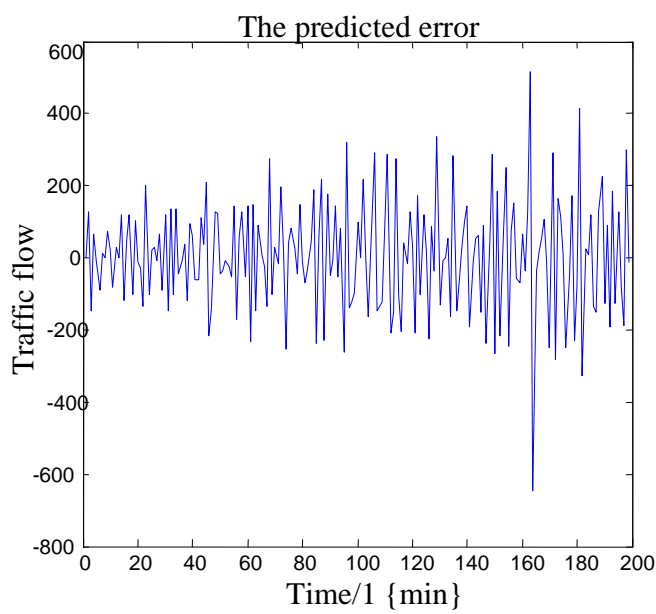

Fig. 6 Single-index moving predicted error

The single-index moving method prediction results and prediction error are shown in figure 5 and 6.The overall trend of prediction is good, however; there exists obvious hysteresis and large deviation in comparison with the real values. The trend by using single-index moving method is pretty reliable which could be utilized as an advantage for further study when combined with other prediction methods.

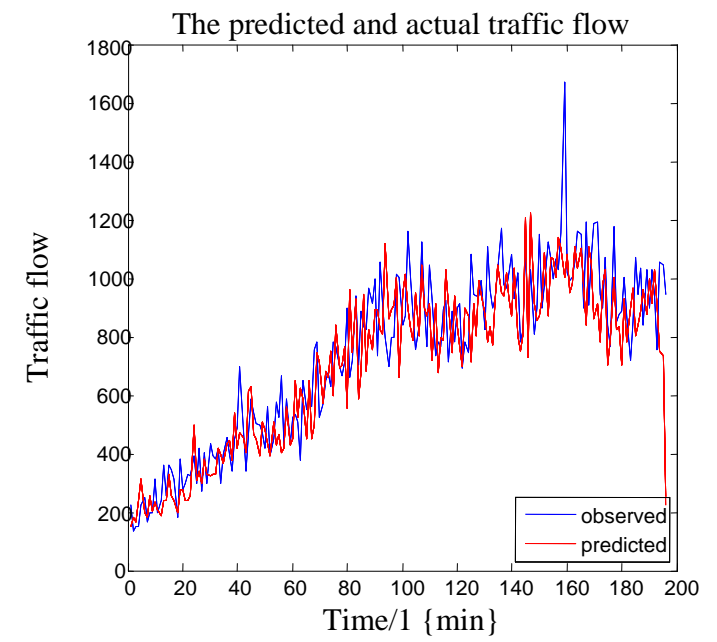

Fig. 7 BP neural network predicted results

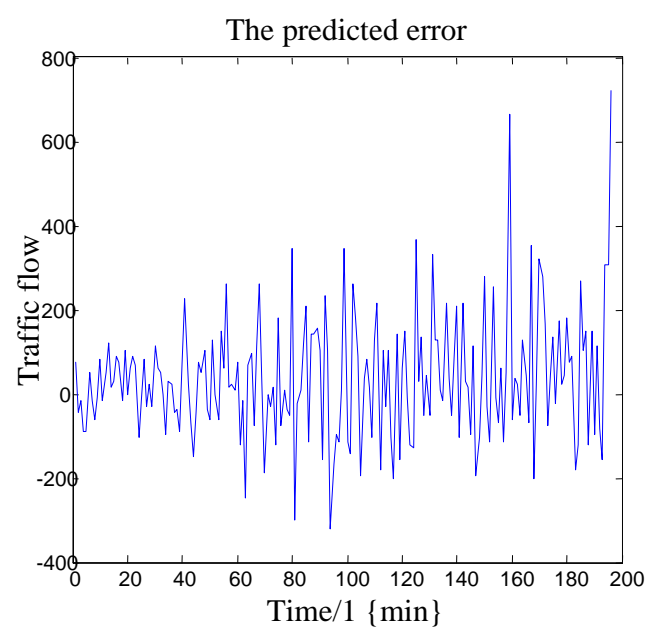

Fig. 8 BP neural network predicted error

The BP neural network prediction results and prediction errors are shown in figure 7 and 8.The predicted results of BP neural network is slightly better than single-index moving method due to the use of the ability of nonlinear mapping and the non-essential to put forward calculation formula and large quantity of mathematical assumptions. The BP neural network also transform the function of tradition into high-dimensional nonlinear mapping. However, its overall trend is not as good as the single-index moving, so that it is still not an ideal prediction method of traffic flow. 


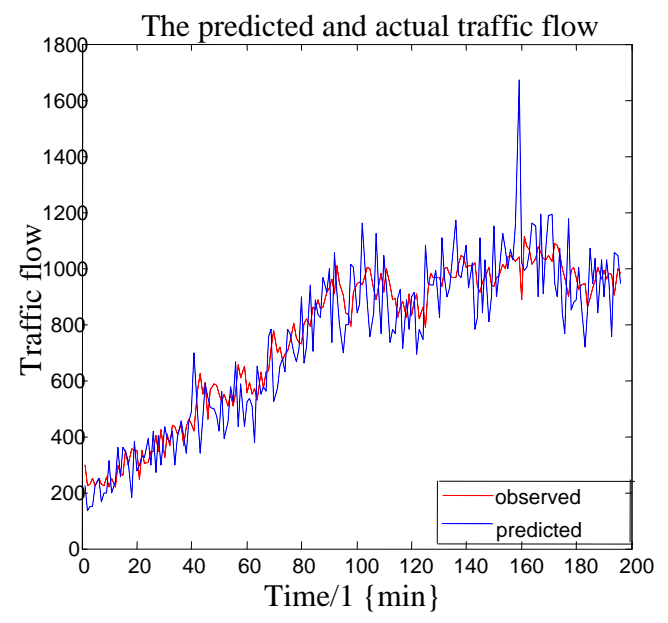

Fig. 9 EMD-WNN predicted results without filtering

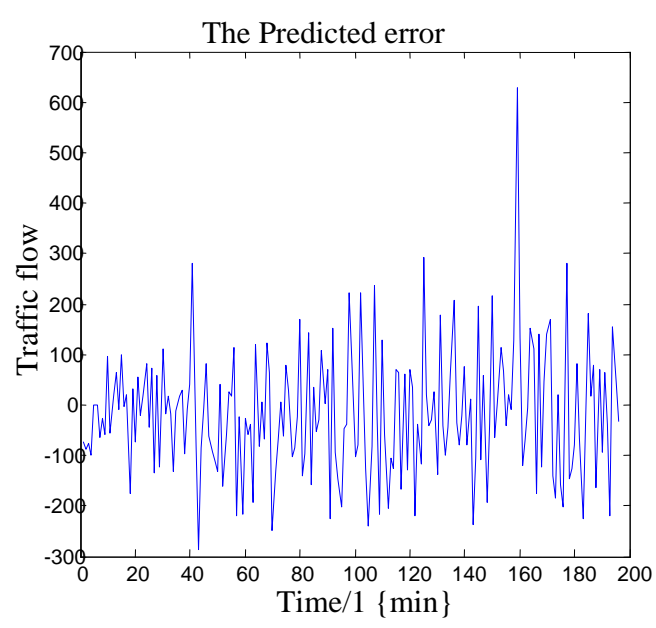

Fig. 10 EMD-WNN predicted error without filtering

Figure 9 is the prediction by accumulating each predicted modal results after processing EMD decomposition of the original traffic flow data. Figure 10 is the corresponding absolute error. Figure 9 and Figure 10 demonstrate that the proposed the EMD-WNN makes a distinct improvement compared with the previous two methods. The predicted effect performs well in both prediction trend and the prediction value. However, the predicted error is still relatively large.
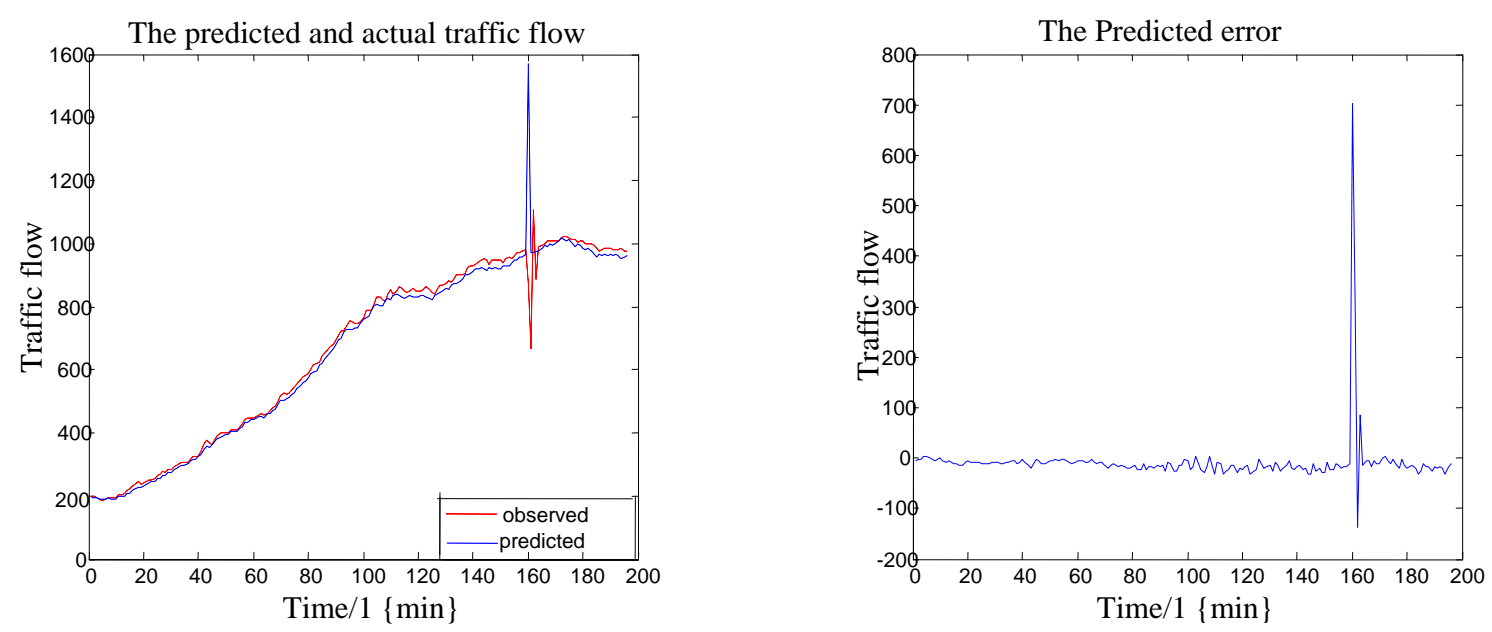

Fig. 11 The predicted results of EMD-WNN with filtering Fig. 12 The predicted error of EMDWNN with filtering

Every modal is analyzed after processing EMD decomposition of the original traffic flow data and the random noise is separated. The modal comes from effective signals are predicted respectively and the result are added afterwards. Figure 11 shows the prediction result while Figure 12 illustrates the corresponding absolute error. Figure 11 and 12 demonstrate that the effect is much better than several previous methods. Error between prediction and actual values is almost zero before the mutated of the traffic. Thus the result is reliable and the method is feasible. Which illustrate the method is fairly accurate and simple and can guide the traffic flow correctly.

In order to assess the effectiveness of the proposed prediction method, the proposed evaluation indexes are calculated and the results are shown in table 1. 
Table 1 The results of data

\begin{tabular}{|c|c|c|c|c|}
\hline Evaluation index & MAE & MSE & MAPE & MSP \\
\hline $\begin{array}{l}\text { The predicted method of } \\
\text { EMD-WNN without filtering }\end{array}$ & 91.9747 & 8.4477 & 0.1432 & 0.0716 \\
\hline $\begin{array}{l}\text { The predicted method of } \\
\text { EMD-WNN with filtering }\end{array}$ & 17.4272 & 4.6758 & 0.0318 & 0.0716 \\
\hline $\begin{array}{l}\text { BP neural network predicted } \\
\text { method }\end{array}$ & 110.9719 & 10.7988 & 0.1551 & 0.0716 \\
\hline $\begin{array}{l}\text { single-index moving method } \\
\text { predicted method }\end{array}$ & 118.9869 & 10.9532 & 0.1842 & 0.0716 \\
\hline
\end{tabular}

Table 2 The results of evaluation

\begin{tabular}{ccccc}
\hline Prediction method & $\begin{array}{c}\text { single-index } \\
\text { moving } \\
\text { method }\end{array}$ & $\begin{array}{c}\text { BP neural } \\
\text { network }\end{array}$ & $\begin{array}{c}\text { method of } \\
\text { EMD-WNN } \\
\text { without } \\
\text { filtering }\end{array}$ & $\begin{array}{c}\text { method of } \\
\text { EMD-WNN } \\
\text { with filtering }\end{array}$ \\
\hline $\begin{array}{c}\text { The results of } \\
\text { evaluation }\end{array}$ & common & common & good & excellent \\
\hline
\end{tabular}

In all, the EMD-WNN method without filtering in the aspect of prediction effect is better than single-index moving method and BP neural network method, however, EMD-WNN prediction method after filtering is performs better. The conclusion could be obtained more intuitive from Table 1 and Table 2. Therefore, the proposed EMD-WNN filtering traffic flow prediction method has rather high effectiveness.

\section{Conclusion}

This paper combines the characteristics of global search ability of genetic algorithm and fast high precision of wavelet neural network. Then a model of wavelet neural network based on genetic algorithm which avoid the low precision of genetic algorithm effectively and the shortcoming of easily trapped in local minimum of wavelet neural network is given. It realized that the high precision global optimization of parameters. The whole traffic flow of urban traffic network could be predicted accurately in a short period after applying this method in the intersections and it has theoretical and practical significance in the further path optimization and traffic route guidance. Moreover, a great support could be provided for real-time traffic management through the analysis of actual data. Meanwhile the improvement of prediction precision and the dealing method of the data spatial relationships of wavelet neural remains further research.

\section{References}

[1] H.Dia:European Journal of Operational Research Vol.37 (2001), p.149-151

[2] S.E.Jabari and H.X. Liu: Transportation Research Vol.46(2013), p.156-174

[3] Chao Yang and Zhiwei Wang:Computer engineering Vol.37(2011), p.149-151

[4] Yuehui Chen,Bin Yang and Qingyang Meng: Applied Soft Computing Journal Vol.12(2011),p.274-279

[5] E.I.Vlahogianni,M.G.Karlaftis and J.C.Golias: Transportation Research Vol.13(2005), p.211234

[6] Xiaoli Zhang and Guoguang HE: Systems Engineering-Theory \& Practice Online Vol.27(2007), p.167-171 